\title{
Überlieferung:
}

Aus Kreuzer, Heinrich, 151-168.

Mit vollständigem Text:

Erfurt, UB, Dep. Erf., CA $4^{\circ} 145,14$. Jh. (Ende), fol. 36r(34r)-66v(63v).

Heiligenkreuz, StiftsB, 290, 14. Jh. (Ende), fol. 114ra-137vb.

Klosterneuburg, StiftsB, 700, 15. Jh., fol. 37ra-60va.

München, BSB, Clm 26608, 15. Jh., fol. 59r-87v.

Paris, BnF, lat. 14644, 14./15. Jh., fol. 142r-161v.

Wolfenbüttel, HAB, 678 Helmstedt, 14. Jh. (Ende), fol. 35r-52r.

Wolfenbüttel, HAB, 264.25 Extravagantes, 14. Jh. (Ende).

Mit unvollständigem Text:

Eichstätt, UB, Cod. st 321, 14./15. Jh., fol. 15ra-37vb.

Innsbruck, UB, 129, 15. Jh., fol. 149ra-159va.

Mainz, StB, II 223, 15. Jh., fol. 147r-167r.

Paris, BnF, lat. 1462, 14. Jh. (Ende), fol. 74r-85v.

Rouen, BM, 1355 (O.20), 14. Jh., fol. 132v-133r (pars 88).

\section{R71. Iohannes Conversinus de Ravenna, 1343-1408 \\ Iohannes Ravennatensis, Giovanni Conversini da Ravenna, Giovanni Conversano, Giovanni di Conversino, Giovanni Da RAVENNA}

Autor: Giovanni Conversini wurde 1343 in Buda geboren. Dort war sein Vater Arzt des ungarischen Königs Ludwig von Anjou. Nach dem frühen Tod der Mutter wurde er nach Ravenna geschickt, wo er unter der Aufsicht seines Onkels Tommaso da Frignano OFM stand. Nach schulischer Bildung in Ravenna studierte er in Bologna, Ferrara und Padova. 1355 heiratete er und ein Jahr später wurde sein erster Sohn, Conversino, geboren. Seine Frau verstarb 1369. 1374 heiratete er zum zweiten Mal in Belluno; 1375 wurde sein Sohn Israele geboren. 1378 wurde sein Onkel Tommaso, inzwischen Patriarch von Grado, zum Kardinal ernannt. Bis zu diesem Zeitpunkt hatte Giovanni wechselnde Stellen als Privatlehrer, Dozent in Florenz oder Lehrer in verschiedenen Städten gehabt und einige kurze philosophische Traktate geschrieben. Mit dem Dialogus inter Fohannem et literam beglückwünscht er seinen Onkel zu seiner Ernennung und bittet ihn, ihm eine dauerhafte Stelle zu suchen. Sein Onkel konnte ihm zwar nicht helfen, aber kurz darauf engagierte ihn Francesco il Vecchio da Carrara, Herrscher von Padua, als Sekretär. Wegen des Todes seiner zweiten Frau und Auseinandersetzungen mit anderen Höflingen verließ er bereits 1383 Padua und eröffnete eine eigene Schule in Venedig. Noch im selben Jahr 
wurde er zum Kanzler der Stadt Ragusa (heute Dubrovnik), die unter ungarischer Herrschaft stand, ernannt. 1387 kehrte er nach Venedig zurück und startete erneut eine eigene Schule. 1389 nahm er eine Stelle als Lehrer in Udine an und wechselte 1392 nach Padua, wo er die nächsten zwölf Jahre blieb. In diesen Jahren unterrichtete er, stand immer wieder im Dienst bei Francesco da Carrara, für den er sogar drei Gesandtschaften übernahm, und verfasste verschiedene Erzählungen, kurze Traktate über das Leben am Hof und eine Autobiographie, das Rationarium vite. 1401 verstarb sein Sohn Israele. 1404 wechselte er von Padua nach Venedig, wo er erneut unterrichtete. Dort entstanden seine Dragmalogia, die Erzählung Conventio inter podagram et araneam und eine Anekdotensammlung, der Memorandarum rerum liber. Giovanni Conversino verstarb vor dem 27. September 1408 in Venedig.

\section{Literatur:}

Kohl, Benjamin G., „The Works of Giovanni di Conversino da Ravenna: a Catalogue of Manuscripts and Editions“, Traditio 31 (1975), 349-367, auch in: Kohl, Culture, Aufsatz IV.

Kohl, Benjamin G., ,,The Manuscript Tradition of Some Works of Giovanni da Ravenna“, in: Tuynman, Pierre / Kuiper, Gerdien C. / Keßler, Eckhard, Acta Conventus Neo-Latini Amstelodamensis, München 1979, 610-619, auch in: Kohl, Culture, Aufsatz V.

Kohl, Benjamin G. „Conversini, Giovanni“, DBI 28 (1983), 574-578.

Kohl, Benjamin G., Culture and Politics in Early Renaissance Padua, Aldershot u. a., 2001.

Leoncini, Letizia, „Forme editoriali del testo e commento figurato. II. Il caso di Giovanni Conversini da Ravenna“, in: Intorno al testo. Tipologie del corredo esegetico e soluzioni editoriali. Atti del convegno di Urbino 1-3 ottobre 2001, Roma 2003, 485-495. Über die Hss. Zagreb, Knjižnica Jugoslavenske Akademije, II.C.61; Oxford, Balliol College, 288; Paris, BnF, Lat. 6494, und Venezia, Biblioteca Querini-Stampalia, Classe IX n. 11.

Sabbadini, Remigio, Giovanni da Ravenna, insigne figura d'humanista (1343-1408), Como 1924 (ND Torino 1961).

\section{R71A. Dialogus INTER IOHANNEM ET LITTERAM}

Rep.: Kohl 4; von Perger 115.1.

Studie: Kapitel 5.3.2.

Widmung: Omnis reverentiae et sanctitatis cultu dignissimo patri domino Thomae, titulato Sanctorum Nerei, Archilei atque Pancracii presbytero cardinali beatissimo, integra subiectione devotissimus servus et affectu plenissimus filius. 
Incipit: Iohannes: Quid agimus, Littera? Merendone tabescemus quod dominum, quod patrem, quod columen unicum spei nostrae procul miserimus?...

Explicit: Littera: En pergo. Tu prosperum interim mihi iter, faustum deinde successum ac reditum superos ora. Vale. Anno imperii domini nostri Jesu Christi millesimo trecentesimo septuagesimo nono indictione secunda nonis ianuariis.

Widmungsverse am Ende des Dialogs: Vertice purpureo sacro qui in cardine mundi / fulge, Thoma, tui pueri dignare volumen. / Quamquam te resonet totum tua fama per orbem, / hoc quoque venturis nostro discere labore.

Datierung: Vom Autor am 5. Januar 1379 datiert (vgl. Explicit). Der Dialogus wurde nach der Ernennung seines Onkels Tommaso da Frignano zum Kardinal am 18. September 1378 geschrieben (vgl. Kohl, „Conversini“", 575).

Inhalt: Dialog zwischen Johannes und Littera, dem Autor und dem Brief, den er an seinen Onkel Tommaso schickt. Littera hat nur kurze Gesprächseinlagen, die dem Autor die Gelegenheit zu ausführlichen Antworten geben. Der Brief befürchtet, nicht aufgenommen zu werden, da er arm ist und die Kirchenmänner geldgierig und geizig. Johannes antwortet darauf mit Lobreden auf seinen Onkel. Der Brief erkundigt sich nach dem Leben des Kardinals und Johannes antwortet mit einer Schilderung des Werdegangs seines Onkels, in der er immer wieder seine Tugenden lobt und passende Anekdoten erzählt. Eine Klage über die Situation Italiens führt zu der Bitte, der Onkel möge in Rom eine passende Stelle für Johannes suchen. Der Brief agiert jetzt als Ankläger und nennt die Lebensumstände, die Johannes nicht erlauben, in den Genuss einer Pfründe zu kommen: seine zwei Eheschließungen und seine zwei Kinder. Dies veranlasst Johannes zu allgemeinen Betrachtungen über die Ehe, die Beziehung zu den eigenen Kindern und die Kindererziehung. Da Johannes keine Pfründe bekommen kann, möchte er irgendeine Stelle, die ihm genug Zeit für seine Studien lässt. Er beklagt seine aktuellen Umstände und preist seine Kenntnisse und Fähigkeiten. Zuletzt gibt er dem Brief ausführliche Anweisungen, wie er sich bei seinem Onkel vorstellen und benehmen soll.

\section{Edition:}

Eaker, Helen Lanneau / Kohl, Benjamin G., Giovanni Conversini da Ravenna. Dialogue between Giovanni and a Letter, New York 1989. Kritische Edition mit englischer Übersetzung von H. L. Eaker, der sparsame Apparat steht nicht unter dem Text, sondern im Anhang (170). Einführung und Kommentar von Eaker und Kohl. 


\section{Literatur:}

Kohl, Benjamin G., „Readers and Owners of an Early Work of Giovanni Conversini da Ravenna: New College, Oxford, ms. D. 155“, Scriptorium 40 (1986), 95-100 und Tafeln 6-7, jetzt auch in: Kohl, Benjamin, Culture, Aufsatz VI.

\section{Überlieferung:}

Aus der Ed. Eaker / Kohl, 14-15:

Oxford, New College, 155 (olim ms. D. 155), 14. Jh. (Ende). Exemplar des Tommasso da Frignano (vgl. Kohl, ,Readers“).

Vaticano, BAV, Pal. lat. 970, 14. Jh. (Ende). Vielleicht Conversinis Exemplar, später in Besitz seines Schülers Francesco Barbaro, der den Text ergänzte und korrigierte.

Wien, ÖNB, 3449, 15. Jh.

Zagreb, Knjižnica Jugoslavenske Akademije Znanosti i Umjetnosti (Akademie der Künste und Wissenschaften), II.C.61, fol. 179v-180r. Fragment aus dem Anfang des Dialogs.

Kohl, „Manuscript tradition..." erfasst auch die Transkription der Hs. Zagreb, die F. Novati Anfang des 20. Jhs. für Remigio Sabbadini anfertigte. Sie wird aufbewahrt in Milano, Società Storica Lombarda, mit der Signatur 43. Es sind zwei Bände, der Dialogus befindet sich in Bd. 2, fol. 125-126.

R71в. DOLOSI ASTUS NARRATIO

Historia Lugi et Conselicis / Dolosa prodictio Lugi et Conselicis domini marchionis

Rep.: Kohl 8; von Perger 115.2.

Studie: Kapitel 5.3.2.

Prolog: Incipit: Quo Lugi et Conselicis oppida Marchioni eventu subducta forent... Explicit:... et liberiori licentia tractantur.

Incipit: Galba: Quod te nunc otium, Cato, sequestrem haec inter frondentia tenet?

Explicit:... tempestas hortatur. Eamus.

Datierung: Der Text basiert auf Vorkommnissen des Jahres 1395 und ist vermutlich nicht viel später entstanden (Sabbadini, 91-93).

Inhalt: Zusammenfassung nach den Angaben bei Sabbadini, 91-93. Dieser Autor gibt die Namen der historischen Personen in der Erzählung nicht an. Dialog zwischen zwei alten Römern, Galba und Cato. Galba erzählt und Cato kommentiert die geschilderten Begebenheiten. 
Es werden folgende historische Ereignisse berichtet. Ferrara wurde 1395 von den Vormündern des kleinen Niccolò III. regiert. Der exilierte Azzo d'Este wollte die Stadt mit Hilfe des Grafen Giovanni von Cunio einnehmen und wieder die Regentschaft übernehmen. Die Vormünder, die einen solchen Versuch fürchteten, schmiedeten den Plan, Azzo umzubringen. Ihr Verbündeter Giovanni de Sangiorgio, ein Kaufmann aus Bologna, bestach den Arzt Antonio, damit er und ein Verwandter des Grafen namens Conselice Azzo d'Este töteten. Als Belohnung würde der Graf einen großen Geldbetrag und zwei Städte, Lugo und Conselice, bekommen. Conselice täuschte Einverständnis vor, setzte aber Azzo und den Grafen vom geplanten Attentat in Kenntnis. Diese ließen einen Diener von Azzo, der seinem Meister ähnelte, die Kleidung Azzos tragen. Conselice tötete ihn und verkündete die Ermordung Azzos. Daraufhin bekam der Graf die versprochenen Städte.

\section{Editionen}

Unediert.

Eine kritische Edition wurde angekündigt:

Albanese, Gabriella / Leoncini, Letizia (Hgg.), Giovanni Conversini da Ravenna. Le novelle della corte Carrarese.

Die Vorrede und der Beginn des Dialogs wurden von Angelo Maria Querini aus der Hs. Vat. lat. 1666 transkribiert und in einem Brief zitiert: „Epistula LXXV ad Joannem Chrysostomum Trombelli" in: Coleti, Nicolaus (Hg.), Epistolae eminentiss. et reverendiss. D. D. Angeli Mariae Quirini (...) quotquot Latino sermone is edidit, quaeque seu seorsim, seu in decades distributae antea vagabantur, Venezia 1761, 565-570, hier 569.

\section{Überlieferung:}

Aus Kohl, „Works of Giovanni“, hier 352-354:

Modena, Biblioteca Capitolare, O. I. 15, 15. Jh., fol. 13r-19r. Titel: Dolosa prodictio Lugi et Conselicis domini marchionis.

Oxford, Balliol College, 288, 15. Jh. (Anfang), fol. 171v-180v.

Vaticano, BAV, Vat. lat. 1666, hier 14. Jh., fol. 28v-38r.

\section{R71c. VIOLATAE PUDICITIAE NARRATIO}

\section{Historia moralis Elysiae}

Rep.: Kohl 9; von Perger 115.3.

Studie: Kapitel 5.3.2.

Prolog: Incipit: Quamquam illustribus iamdudum pateat exemplis... Explicit:... Damone Pithiaque notis nominibus tractatur ipsa narratio. 
Incipit: Damon. Interfuisses historiae, quam nuper audivi, ita pudicitiae pretio affectus esses...

Explicit:... Coniugii foedus oratione elegantissima sancit, una repatrient iubet.

Datierung: Sabbadini, 93, datiert diesen Text in den zweiten Aufenthalt Conversinis in Padua, 1392-1404. Die Historia ist vermutlich um dieselbe Zeit entstanden wie die Dolosi astus narratio, um 1396 (Sabbadini, 93).

Inhalt: Dialog zwischen Damon und Pithias, Damon berichtet, Pithias stellt Fragen, damit Details präzisiert und Hintergründe erklärt werden, und kommentiert die Geschehnisse. Folgende Geschichte wird erzählt: Der französische Ritter Henricus will in das Heilige Land reisen. Bevor er aufbricht, vertraut er sein Haus und seine Frau Elysia seinem Freund Arnaldus an. Arnaldus verliebt sich in Elysia, sucht eine Gelegenheit, mit ihr allein zu sein, und vergewaltigt sie. Elysia vertraut sich niemandem an. Um ihren Kummer zu erklären, erzählt sie, sie habe vom Tod ihres Mannes geträumt. Als ihr Mann zurückkommt, erzählt sie ihm die Wahrheit. Henricus klagt Arnaldus vor dem König an, aber Arnaldus streitet die Anschuldigung ab. Es kommt zu einem Gottesgericht, bei dem es zuerst so aussieht, als würde Arnaldus siegen, er fällt jedoch auf Henricus' Messer hin, verletzt sich schwer und stirbt. Henricus und Elysia werden am Hof erneut vermählt.

\section{Editionen}

Korelin, Michail S., Rannij ital'janskij gumanizm i ego istoriografija, Moskva 1892, appendix vi, 16-26, unvollständiger Text nach der Handschrift in der BAV (nur in dieser Auflage, nicht mehr in der zweiten Auflage, St. Petersburg 1914).

Wolfer, Isabel, „Giovanni Conversini da Ravenna (1343-1408): Violate pudicicie narratio sive Historia Elysie", Neulateinisches Fahrbuch 8 (2006), 313-365. Kritische Edition mit deutscher Übersetzung und Anmerkungen.

Eine weitere kritische Edition wurde angekündigt (vgl. Leoncini, „La novella"):

Albanese, Gabriella / Leoncini, Letizia (Hgg.), Giovanni Conversini da Ravenna. Le novelle della corte Carrarese.

Die Vorrede und ersten Zeilen des Dialogs wurden aus der Hs. Vat. lat. 1666 von Angelo Maria Querini transkribiert und in einem Brief zitiert: „Epistula LXXV ad Joannem Chrysostomum Trombelli“ in: Coleti, Nicolaus (Hg.), Epistolae eminentiss. et reverendiss. D. D. Angeli Mariae Quirini (...) quotquot Latino sermone is edidit, quaeque seu seorsim, seu in decades distributae antea vagabantur, Venezia 1761, 565-570, hier 568-569. 


\section{Literatur:}

Leoncini, Letizia, „La novella a corte: Giovanni Conversini da Ravenna“, in: Albanese, Gabriella / Battaglia Ricci, Lucia / Bessi, Rossella (Hgg.), Favole, parabole, istorie. Le forme della scrittura novellistica dal Medioevo al Rinascimento. Atti del Convegno di Pisa 26-28 ottobre 1998, Roma 2000, 189-222.

\section{Überlieferung:}

Aus Kohl, „Works of Giovanni“, 351-354 (Beschreibungen der Hss. in Ed. Wolfer, 323-326):

Oxford, Balliol College, 288, 15. Jh. (Anfang), fol. 165v-171v. Titel: Violate pudicicie narracio Fohannis da Ravenna collocutores Damon et Pithias.

Vaticano, BAV, Vat. lat. 1666, hier 14.Jh., fol. 23r-28v. Praescriptio: Johannis de Ravena historici et moralis Elysie incipit historia.

Venezia, BNM, Lat. XIV. 224 (= 4341), 15. Jh. (Anfang), fol. 129ra-132vb. Titel: Violate pudicicie narracio fo. de Ra. collocutores Pithias: Damon.

Aus Ed. Wolfer, 321-323:

Firenze, BNC, II. I. 64, 15. Jh., fol. 64r-67r.

R71D. DE CONSOLATIONE DE OBITU FILII

Rep.: Kohl 15.

Studie: Kapitel 5.3.2.

Incipit: Immo contumacies [sic] non possint... [Angabe bei Kohl]; Maestus: Heu, heu me fili miserum morte tua... [Hs. Oxford].

Explicit:... dolens retinens meliora. M CCCCI VIII kalendarum Octobrium Patavii [Hs. Oxford].

Datierung: In der Oxforder Handschrift, fol. 71v, ist das Werk auf den 24. September 1401 datiert. Der erwähnte Tod seines Sohnes Israele ereignete sich im Sommer dieses Jahres.

Inhalt: Zusammenfassung nach den Angaben bei Sabbadini, 87-88. Dialog zwischen Mestus, der den Tod des Sohnes betrauert, und Solator. Mestus erzählt die Lebensgeschichte seines Sohnes Israele, der 1375 geboren wurde. Israele war lebhaft und unruhig. Mehrmals floh er von der Schule, was ihn in Schwierigkeiten brachte. Bei einem Fluchtversuch stieß er in den Bergen auf Wölfe. Bei einem anderen Fluchtunternehmen versuchte er, über die Berge zu kommen, und lief Gefahr, von den Wallachen gefangen und als Sklave verkauft zu werden. Er wurde noch rechtzeitig von Kaufleuten gefunden. Als er in Padua die Dialektik-Kurse besuchte, flohen alle Schüler gemeinsam und kamen bis Treviso, wo sie von Paolo Rugulo festgenommen wurden. 
Das war allerdings der letzte Ausbruch von Israele, der danach seine Einstellung änderte, woraufhin der Vater ihn nach Padua zur Artistenfakultät schickte. Solator versucht Mestus mit zahlreichen Zitaten und Beispielen aus klassischer und christlicher Literatur zu trösten.

McClure, der den Text in der Oxforder Handschrift gelesen hat, gibt weitere Informationen zum Inhalt und zur Dialogform:

Like Manetti, Conversini used the dialogue to define and resolve grief. His interlocutors, however, do not have the focused and sustained ,adversarial“" exchange found in the debate between Salutati and Zabarella or in that between „Manetti“ and „Acciaiuoli“. In fact, the speakers Mestus (the bereaved) and Solator (the consoler) do not exclusively perform the roles their names would suggest. Although Solator often attempts to console Mestus, sometimes the reverse occurs. The principal function of the dialogue is to provide a framework for Conversini's attempt to express, dispute, and resolve certain aspects of his bereavement. The literary speakers give voice to an inward colloquy of emotional, philosophical, and spiritual issues - among them, the legitimacy of grief; the character and history of the child; the irreparability and particular pain, frustration, and disappointment of losing a child; the nature of life and death; the loss of a continuator and heir; the acceptance of divine will; the nature of heavenly beatitude; the continuation of ,ties“ between the living and the dead. In its structure the work illustrates again the humanists' experimental use of rhetoric to deal with emotional experience. Through a regimen of debate, remembrance, and rumination, Conversini too gave quarter to his sorrow by fashioning a literary framework for mourning. (McClure, Sorrowe, 104).

\section{Editionen:}

Unediert bis auf Exzerpte bei:

Sabbadini, Remigio, Giovanni da Ravenna, insigne figura d'humanista (1343-1408), Como 1924, 174-176.

\section{Literatur:}

McClure, George W., ,,The Art of Mourning: Autobiographical Writings on the Loss of a Son in Italian Humanist Thought (1400-1461)", Renaissance Quarterly 39 (1986), 440-475.

McClure, George W., Sorrow and Consolation in Italian Humanism, Princeton 1990, hier 93-115.

\section{Überlieferung:}

Aus Kohl, „Works of Giovanni“, 351-353:

Oxford, Balliol College, 288, 15. Jh. (Anfang), fol. 54v-71v.

Venezia, BNM, Lat. XIV. 224 (= 4341), 15. Jh. (Anfang), fol. 134-138. Unvollständig, nur der Schluss. 
Rep.: Kohl 17; RepFont 5, 138; von Perger 115.4.

Studie: Kapitel 5.3.2.

Incipit: Venetus: Advenire te sospitem, Paduane exoptate dudum atque amate, plurimum grator.

Explicit: Paduanus: Tuo parerem arbitratu nisi procedere iuberet. Bene vale. Venetiis anno Domini MCCCCIIII.

Datierung: 1404 (vgl. Explicit).

Inhalt: Dialog zwischen Venetianus und Paduanus. Der Dialog beginnt mit der freundschaftlichen Begrüßung beider. Einigen Aussagen im Dialog ist zu entnehmen, dass beide gemeinsam auf Reisen sind. Nach dem kurzen Gruß kommen sie sofort auf den Krieg zwischen Padua und Venedig zu sprechen. Sie unterhalten sich über den neuen Fürsten von Padua, Francesco Novello da Carrara und seine Einstellung gegenüber Literaten, wobei ein Ravennas (Giovanni Conversino) erwähnt wird. Sie besprechen das Schicksal des Giovanni bei Hof und die Eigenschaften, die ein Höfling braucht, um zu bestehen. Die Gesprächspartner diskutieren anschließend, ob Monarchie oder Republik die überlegene Regierungsform sei. Das nächste Thema ist der Unterschied zwischen den Anreden dominus und magister, und welche der beiden vorzuziehen sei, wobei der Gegensatz zwischen einem aktiven und einem kontemplativen Leben diskutiert wird. Das bringt die Freunde dazu, das Leben in der Stadt und das Leben auf dem Lande zu vergleichen. Da der Paduaner bei seinem Lobpreis des Landes die Armut rühmt, überlegt er, dass manchmal ein scheinbares Übel gut sein kann. Das Gespräch wird zu einer Reflexion über das Böse und seine Rechtfertigung, in dem auch die sieben Todsünden analysiert werden, um aufzuzeigen, dass sogar sie manchmal positive Folgen haben können. Der Paduaner kritisiert die Stadt Venedig. Der Venezianer verteidigt seine Landsleute, indem er erklärt, dass sie die Freiheit über alles lieben. Die Diskussion kreist dann um die Freiheit und ihre Bedeutung, vor allem über ihre moralische Dimension, als Unabhängigkeit von Materiellem. Das bringt das Gespräch auf Kardinal Tommaso da Frignano, den Onkel Conversinis, mit dessen Lob der Dialog endet. Im Dialog hat der Paduaner eindeutig eine überlegene Stellung, er lenkt das Gespräch und hält ausführliche Reden, auf die der Venezianer meistens nur kurz reagiert. In der Diskussion kann der Paduaner seine Ansichten durchsetzen: 